\title{
Cératopogonidés
}

\section{de la côte de Pologne}

\author{
par Barbara SKIERSKA \\ Laboratoire d'Entomologie médicale,' Chaire de Parasitologie tropicale \\ de l'Institut de la Médecine maritime de Gdansk, Pologne \\ incs
}

\section{Résumé.}

En se basant sur les recherches de quelques années, on a constaté la présence habituelle de Cératopogonidés hématophages dans les terrains côtiers. On a défini les types des gîtes larvaires, on a examiné les conditions du développement des larves dans les gîtes salés, ainsi que le degré et les conditions d'agressivité des femelles aux appâts humains et animaux.

\section{Summary.}

\section{Ceratopogonidae from the Polish coast.}

On the base of some year researches, we have found the usual presence of hematophagous Ceratopogonides in the coastal fields. We defined the larval breeding places, we examined the developmental conditions of the larvae in the Salt marsh breeding places, and the degree and conditions of aggressivity of the females to human and animal baits.

Les recherches de quelques années sur les Cératopogonidés hématophages de la côte de Pologne ont montré que dans les terrains de l'estuaire de la Vistule et de l'Odra ces insectes apparaissent habituellement.

Les investigations étaient effectuées sur les différents biotopes caractéristiques de ces terrains (forêts côtières sur les dunes, forêts d'aulnes humides sur les bords des lacs, prés, pâturages et marécages salés).

Les Cératopogonidés étaient obtenus à partir de récoltes de boues de gîtes larvaires et par élevage de larves ainsi que par des captures de femelles agressives sur l'homme et les animaux domestiques (les vaches).

Les résultats des recherches ont permis de définir la composition faunistique (tableau I) et la phénologie de la population des Cératopogonidés hématophages, de déterminer des types des gîtes larvaires - particulièrement les conditions du développement des larves dans les gîtes salés (fig. 1,2) - ainsi que le degré et les conditions d'agressivité des femelles. 
Sur la base des observations qui étaient faites systématiquement chaque semaine, depuis le mois de mai jusqu'au mois d'octobre on a constaté que les femelles hématophages des Culicoïdes sp. ont montré l'agressivité la plus haute dans les conditions météorologiques déterminées, c'est-à-dire à une température de l'air de 12$22,2{ }^{\circ} \mathrm{C}$, une humidité relative de l'air $62,2-95 \%$, une intensité de la lumière 844230 lux, et en l'absence du vent. Le vent plus fort que $3 \mathrm{~m} / \mathrm{sec}$., ainsi que la température de l'air plus élevée que $22,2^{\circ} \mathrm{C}$ ou plus basse que $8,4^{\circ} \mathrm{C}$ et l'humidité relative de l'air plus basse que $50 \%$, ont arrêté totalement l'agressivité des Culicoïdes.

Une fois seulement on a ramassé sur l'homme sept femelles de ce genre (3 $C$. punctatus et $4 \mathrm{C}$. obsoletus) à la température de l'air de $4{ }^{\circ} \mathrm{C}$.

En comparant le pourcentage des groupes d'espèces obtenus par les matériels d'élevage des larves par rapport aux captures des femelles agressives, on a pu constater que dans les conditions examinées sur la côte, les espèces Culicö̈des sp. les plus agressives étaient du groupe obsoletus $(0,011 \%$ des larves et $39,86 \%$ des femelles agressives), ensuite du groupe pulicaris $(5,75 \%$ et $25,53 \%)$ et du groupe fascipennis $(8,08 \%$ et $15,56 \%)$.

Au contraire, les espèces du groupe salinarius qui a donné 78,77 \% des larves, n'étaient pas retrouvées dans les captures d'appât humain et animal.

Dans les observations de laboratoire on a constaté que les femelles $C$. machardyi développent leurs premiers œufs autogéniquement sans prendre de sang.

Les sections d'ovarioles ont montré que les femelles des nombreuses espèces de Culicoïdes sp. dans les conditions examinées avaient passé quatre cycles gonotrophiques, ce qui leur donne la possibilité de transmettre des agents pathogènes.

\section{TABleau I. - Les espèces constatées}

1. Culicoides achrayi Kettl., Law. (1, 2).

2. Culicoides albicans (Winn.) (1).

3. Culicoides chiopterus (Meig.) (2).

4. Culicoides circumscriptus Kieff. (1).

5. Culicoides cubitalis Edw. $(1,2)$.

6. Culicoides duddingstoni Kettl., Law. (1).

7. Culicoides delta Edw. (1, 2).

8. Culicoides fascipennis (Staeg.) (1, 2).

9. Culicoides grisescens Edw. $(1,2)$.

10. Culicoides halophilus Kieff. (1, 2).

11. Culicoides heliophilus Edw. $(1,2)$.

12. Culicoides impunctatus Goetgh. $(1,2)$.

13. Culicoides lupicaris Down., Kettl. (2).

14. Culicoides machardyi C.P.-Cl. (1).

15. Culicoides maritimus Kieff. (1).

16. Culicoides nubeculosus Meig. (1, 2).

17. Culicoides obsoletus Meig. (1, 2).

18. Culicoides odibilis Aust. $(1,2)$.

19. Culicoides pumilus Winn. (2).

(1) Par élevage larvaire.

(2) Par récoltes des femelles agressives.
20. Culicoides pallidicornis Kieff. (1, 2).

21. Culicoides pictipennis Staeg. (1).

22. Culicoides picturatus $\mathrm{Kr}$., Ded. (1).

23. Culicoides pulicaris L. $(1,2)$.

24. Culicoides punctatus (Meig.) (1, 2).

25. Culicoides puncticollis (Beck.) (1).

26. Culicoides reconditus C.P.-Cl. $(1,2)$.

27. Culicoides riethi $\operatorname{Kieff}(1,2)$.

28. Culicoides salinarius Kieff. (1).

29. Culicoides segnis C.P.-Cl. (1, 2).

30. Culicoides simulator Edw. (1).

31. Culicoides. stigma Meig. (1, 2).

32. Culicoides subfascipennis analis Kieff. (1, 2).

33. Culicoides tentorius Aust. (1, 2).

34. Culicoides vexans Staeg. (1).

35. Culicoides vistulensis Skierska (1).

36. Forcipomyia bipunctata (L.) (2).

37. Atrichopogon lucorum (Meig.) (2). 


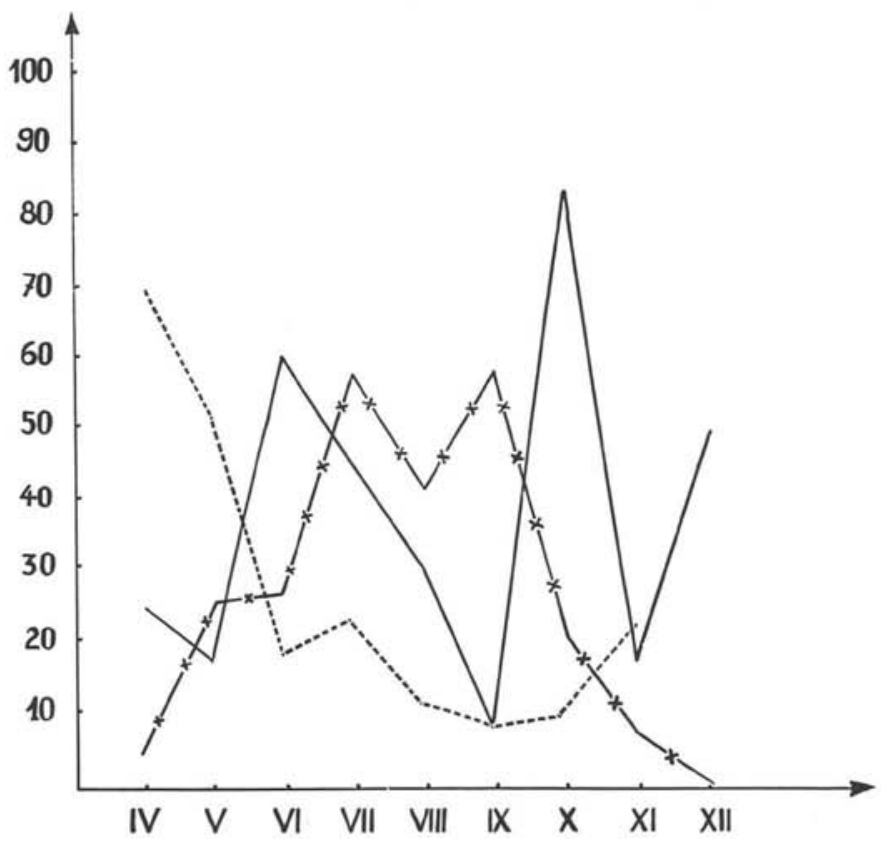

Fig. 1. - Variations saisonnières de la production larvaire de la boue ramassée dans les différents gîtes salés (les moyennes de 3 années).

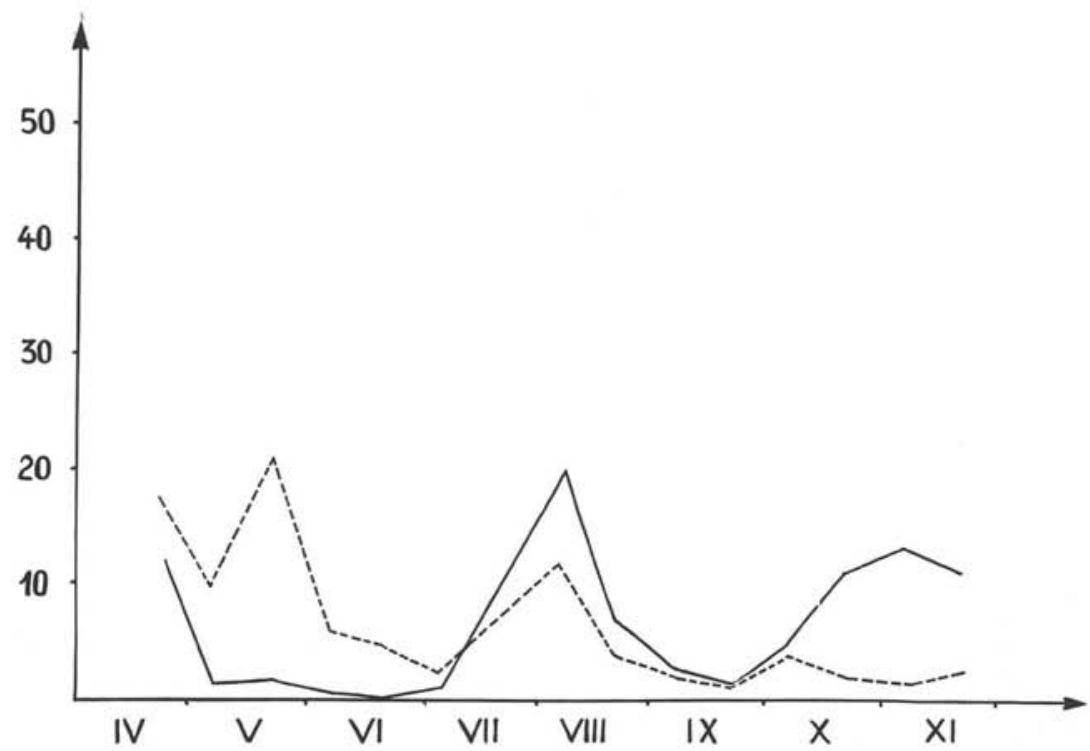

FIG. 2. - Variations saisonnières du nombre de larves de C. salinarius (-) et C. machardyi (--) récoltées dans les gîtes salés de la côte près de Gdansk. 